\title{
Dynamically consistent optical flow estimation
}

\author{
Nicolas Papadakis ${ }^{1}$, Thomas Corpetti ${ }^{1,2}$ and Etienne Mémin ${ }^{1,3,4}$ \\ ${ }^{1}$ IRISA / INRIA, Campus Universitaire de Beaulieu, 35042 Rennes Cedex, France \\ ${ }^{2}$ CNRS / COSTEL UMR 6554, Place du Recteur Henri Le Moal, 35043 Rennes Cedex, France \\ ${ }^{3}$ CEFIMAS, Avenida Santa Fe 1145 C1059ABF - Buenos Aires, Argentina \\ ${ }^{4}$ Fac. de Ing. de la Univ. Buenos-Aires, Av. Paseo Colón 850, C1063ACV Buenos Aires, Argentina \\ \{npapadak, corpetti,memin\}@irisa.fr
}

\begin{abstract}
In this paper, we present a framework for dynamic consistent estimation of dense motion fields over a sequence of images. The originality of the approach is to exploit recipes related to optimal control theory. This setup allows performing the estimation of an unknown state function according to a given dynamical model and to noisy and incomplete measurements. The overall process is formalized through the minimization of a global spatio-temporal cost functional w.r.t the complete sequence of motion fields. The minimization is handled considering an adjoint formulation. The resulting scheme consists in iterating a forward integration of the evolution model and a backward integration of the adjoint evolution model guided by a discrepancy measurement between the state variable and the available noisy observations. Such an approach allows us to cope with several delicate situations (such as the absence of data) which are not well managed with usual estimators.
\end{abstract}

\section{Introduction}

Optical flow estimation is one of the key problems in computer vision and has largely been studied the last two decades since the seminal work of Horn \& Schunck [14]. From the panel of available techniques, the variational methods have been proved to be very efficient to extract accurate instantaneous velocity measurements $[1,4,5,6$, 22, 23, 26, 29]. Performance evaluations of some of these methods can be found in [2,9].

However, the computation of the optical flow over a complete sequence of images remains a difficult problem if one wishes explicitly to maintain a relevant global spatiotemporal coherence. Some spatio-temporal estimators have been proposed in previous studies [4, 5, 22, 29]. Nevertheless, except the two frames Stockes regularization of [27], none of them introduce an explicit dynamic law as a temporal consistency. As a matter of fact, spatio-temporal regularizers as introduced in $[4,29]$ only consider a crude sta- tionary local prior. Moreover, strong artifacts are managed with difficulty. Such approach can not be used to enforce on the whole sequence a solution minimizing an image based discrepancy measure and in the same time that follows a given dynamical model. This kind of tracking process is indeed very difficult to manage with stochastic techniques as the variable's state space is of huge dimension (theoretically infinite) and can not be efficiently handled with usual recursive Bayesian filters such as the particle filter.

In this paper, we propose a variational technique which allows us to estimate a sequence of dense motion fields guided by a given dynamical law. To that end, we suggest to rely on recipes related to optimal control theory $[19,17]$ and variational data assimilation [17] . As Bayesian smoothing, such techniques allow to estimate on the basis of noisy and possibly incomplete observations a feature trajectory (a sequence of dense motion fields in our application) respecting a specified evolution law. The associated minimization process is efficiently expressed considering an adjoint formulation. The adjoint variable introduced enables to compute the gradient of the cost-function from a forward-backward integration of two coupled evolution models. This efficient procedure authorizes coping with state space of very large dimensions.

This framework has been used for two different applications of motion estimation. For each applications the image observations and the dynamical law have been specifically adapted.

In the section 2, we present the general principles of optimal control theory on which this setup relies. In section 3 , we describe its application for the computation of dense motion fields in two different applications involving dynamics of different natures.

\section{Optimal Control Theory}

\subsection{Mathematical formulation}

The problem we are dealing with consists in recovering a system's state $\mathcal{X}(\boldsymbol{x}, t)$ obeying to a dynamical law given 
some noisy and possibly incomplete measurements of the state. The measurements (also called observations) are assumed to be available only at discrete time. This is formalized, for any location $\boldsymbol{x}$ at time $t \in\left[t_{0}, t_{f}\right]$, by the system:

$$
\begin{array}{r}
\frac{\partial \mathcal{X}}{\partial t}(\boldsymbol{x}, t)+\mathbb{M}(\mathcal{X}(\boldsymbol{x}, t), u(t))=0 \\
\mathcal{X}\left(\boldsymbol{x}, t_{0}\right)=\mathcal{X}_{0}(\boldsymbol{x})+\boldsymbol{\epsilon}_{n}(\boldsymbol{x}),
\end{array}
$$

where $\mathbb{M}$ is a non-linear dynamic operator depending on a control parameter $u(t)$. We assume here that $u(t) \in U$ and $\boldsymbol{X}(t) \in \mathcal{V}$ are square integrable functions in Hilbert spaces identified to their dual. The term $\boldsymbol{X}_{0}$ is the initial vector at time $t_{0}$ and $\epsilon_{n}$ is an (unknown) additive control variable on the initial condition. Besides, we assume that measurements of the unknown state $\mathcal{Y} \in \mathcal{O}$ are available. These observations are measured through the non-linear operator $\mathbb{H}$ and belong also to an Hilbert space .

\subsection{Cost function}

The purpose consists in finding a controls of lower energy that leads to the lowest discrepancy between the measurements and the state variable. This can be expressed through the minimization of the following cost function: $\mathcal{J}: U \times \mathcal{V} \rightarrow \mathbb{R}$ as: $\mathcal{J}: U \times \mathcal{V} \rightarrow \mathbb{R}$ as:

$$
\begin{aligned}
\mathcal{J}\left(u, \boldsymbol{\epsilon}_{n}\right) & =\frac{1}{2} \int_{t_{0}}^{t_{f}}\left\|\mathcal{Y}-\mathbb{H}\left(\mathcal{X}\left(u(t), \boldsymbol{\epsilon}_{n}, t\right)\right)\right\|_{R^{-1}}^{2} d t \\
& +\frac{1}{2}\left\|\boldsymbol{\epsilon}_{n}\right\|_{B^{-1}}^{2}+\frac{1}{2} \int_{t_{0}}^{t_{f}}\left\|u(t)-u_{0}\right\|_{F^{-1}}^{2} d t
\end{aligned}
$$

where $u_{0}$ is some expected value of the parameter. The norms $\|\cdot\|_{R^{-1}},\|\cdot\|_{B^{-1}}$ and $\|\cdot\|_{F^{-1}}$ are induced norms of the inner products $<R^{-1} ., .>_{\mathcal{O}},<B^{-1} ., .>_{\mathcal{V}}$ and $<F^{-1}$.,. $>_{U}$ associated to Hilbert spaces $\mathcal{O}, \mathcal{V}, U$ of the measurements, the state variable and the control variable respectively; $R, B$ and $F$ are endomorphism (called covariance matrices) of the observation space, state space and control space. They are related respectively to the observations, the initial condition of the state variable and to the expected value of the control. In our applications, these covariance matrices have been defined as diagonal matrices.

In order to compute the gradient of this functional we assume that $\boldsymbol{X}\left(u(t), \boldsymbol{\epsilon}_{n} ; t\right)$ depends continuously on $u(t), \boldsymbol{\epsilon}_{n}$ and is differentiable with respect to the control variables $\left.u(t), \boldsymbol{\epsilon}_{n}, \forall t \in\right] t_{0}, t_{f}[$.

\subsection{Differentiation}

Noting that $d \mathcal{X}=(\partial \mathcal{X} / \partial u) \delta u(t)+\left(\partial \mathcal{X} / \partial \boldsymbol{\epsilon}_{n}\right) \delta \boldsymbol{\epsilon}_{n} \in$ $\mathcal{V}$, the differentiation of equations (1-2) in the direction $\left(\delta u, \delta \boldsymbol{\epsilon}_{n}\right)$ reads:

$$
\begin{array}{r}
\frac{\partial d \mathcal{X}}{\partial t}+\partial_{\mathcal{X}} \mathbb{M}(\mathcal{X}, u(t)) d \mathcal{X}+\partial_{u} \mathbb{M}(\mathcal{X}, u(t)) \delta u(t)=0, \\
d \mathcal{X}\left(\boldsymbol{x}, t_{0}\right)=\delta \boldsymbol{\epsilon}_{n}(\boldsymbol{x})
\end{array}
$$

where $\partial_{\mathcal{X}} \mathbb{M}$ and $\partial_{u} \mathbb{M}$ are the linear tangent operators defined by:

$$
\lim _{\beta \rightarrow 0} \frac{d \mathbb{M}(\mathcal{X}+\beta \theta, u(t))}{d \beta}=\partial_{\mathcal{X}} \mathbb{M}(\theta)
$$

The differentiation of the cost function (3) in the direction $\left(\delta u, \delta \boldsymbol{\epsilon}_{n}\right)$ (omitting the spatial coordinates $\boldsymbol{x}$ and denoting $U_{T}$ as the space $L^{2}\left(t_{0}, t_{f} ; U\right)$ for sake of clarity) then reads:

$$
\begin{aligned}
& \left\langle\frac{\partial \mathcal{J}}{\partial u}, \delta u\right\rangle_{U_{T}}=\int_{t_{0}}^{t_{f}}\left\langle u(t)-u_{0}, \delta u(t)\right\rangle_{F} d t \\
& -\int_{t_{0}}^{t_{f}}\left\langle\mathcal{Y}(t)-\mathbb{H}(\mathcal{X}(t)),(\partial \mathcal{X} \mathbb{H})\left(\frac{\partial \mathcal{X}}{\partial u} \delta u(t)\right)\right\rangle_{\mathcal{O}} d t \\
& \left\langle\frac{\partial \mathcal{J}}{\partial \boldsymbol{\epsilon}_{n}}, \delta \boldsymbol{\epsilon}_{n}\right\rangle_{\mathcal{V}}=\left\langle\left(\mathcal{X}\left(\boldsymbol{x}, t_{0}\right)-\mathcal{X}_{0}(\boldsymbol{x})\right), \delta \boldsymbol{\epsilon}_{n}\right\rangle_{B} \\
& -\int_{t_{0}}^{t_{f}}\left\langle\mathcal{Y}(t)-\mathbb{H}(\mathcal{X}(t)),(\partial \mathcal{X} \mathbb{H})\left(\frac{\partial \mathcal{X}}{\partial \boldsymbol{\epsilon}_{n}} \delta \boldsymbol{\epsilon}_{n}\right)\right\rangle_{\mathcal{O}} d t
\end{aligned}
$$

These two relations can be reformulated as:

$$
\begin{aligned}
& \left\langle\frac{\partial \mathcal{J}}{\partial u}, \delta u\right\rangle_{U_{T}}=\int_{t_{0}}^{t_{f}}\left\langle F^{-1}\left(u(t)-u_{0}\right), \delta u(t)\right\rangle_{U} d t \\
& -\int_{t_{0}}^{t_{f}}\left\langle(\partial \mathcal{X} \mathbb{H})^{*} R^{-1}\left(\mathcal{Y}(t)-\mathbb{H}(\mathcal{X}(t)), \frac{\partial \mathcal{X}}{\partial u} \delta u(t)\right\rangle_{\mathcal{V}} d t\right. \\
& \left\langle\frac{\partial \mathcal{J}}{\partial \boldsymbol{\epsilon}_{n}}, \delta \boldsymbol{\epsilon}_{n}\right\rangle_{\mathcal{V}}=\left\langle B^{-1}\left(\mathcal{X}\left(\boldsymbol{x}, t_{0}\right)-\mathcal{X}_{0}(\boldsymbol{x})\right), \delta \boldsymbol{\epsilon}_{n}\right\rangle_{\mathcal{V}} \\
& -\int_{t_{0}}^{t_{f}}\left\langle(\partial \mathcal{X} \mathbb{H})^{*} R^{-1}\left(\mathcal{Y}(t)-\mathbb{H}(\mathcal{X}(t)), \frac{\partial \mathcal{X}}{\partial \boldsymbol{\epsilon}_{n}} \delta \boldsymbol{\epsilon}_{n}\right\rangle_{\mathcal{V}} d t\right.
\end{aligned}
$$

where we have introduced the adjoint operator $\left(\partial_{\mathcal{X}} \mathbb{H}\right)^{*}$ defined as:

$$
\forall(x, y) \in(\mathcal{V}, \mathcal{O}),<\left(\partial_{\mathcal{X}} \mathbb{H}\right) x, y>_{\mathcal{O}}=<x,\left(\partial_{\mathcal{X}} \mathbb{H}\right)^{*} y>_{\mathcal{V}}
$$

Expression (7) gives the gradient of the functional in the directions $\left(\delta u, \delta \boldsymbol{\epsilon}_{m}\right)$. We can remark from these expressions that a direct numerical evaluation of these gradients is in practice completely unfeasible. As a matter of fact, such an evaluation would require to compute perturbations of the state variables along all the components of the control variables $\left(\delta u, \delta \boldsymbol{\epsilon}_{n}\right)$ - i.e. integrate our dynamical model for all perturbed components of the control variables which is computationally completely unrealistic.

\subsection{Adjoint model}

An elegant solution of this problem consists in relying on an adjoint formulation [17, 19]. To that end, relation (4) is multiplied by an adjoint variable $\lambda \in \mathcal{V}_{T}$ and integrated over the range $\left[t_{0}, t_{f}\right]$ :

$$
\begin{aligned}
& \int_{t_{0}}^{t_{f}}\left\langle\frac{\partial d \mathcal{X}}{\partial t}(t), \boldsymbol{\lambda}(t)\right\rangle_{\mathcal{V}} d t+\int_{t_{0}}^{t_{f}}\left\langle\left(\partial_{\mathcal{X}} \mathbb{M}\right) d \mathcal{X}(t), \boldsymbol{\lambda}(t)\right\rangle_{\mathcal{V}} d t \\
& +\int_{t_{0}}^{t_{f}}\left\langle\left(\partial_{u} \mathbb{M}\right) \delta u(t), \boldsymbol{\lambda}(t)\right\rangle_{\mathcal{V}} d t=0 .
\end{aligned}
$$


An integration by parts of the first term yields:

$$
\begin{aligned}
& -\int_{t_{0}}^{t_{f}}\left\langle-\frac{\partial \boldsymbol{\lambda}}{\partial t}(t)+\left(\partial_{\mathcal{X}} \mathbb{M}\right)^{*} \boldsymbol{\lambda}(t), d \boldsymbol{X}(t)\right\rangle_{\mathcal{V}} d t=\left\langle\boldsymbol{\lambda}\left(t_{f}\right), d \boldsymbol{X}\left(t_{f}\right)\right\rangle_{\mathcal{V}} \\
& -\left\langle\boldsymbol{\lambda}\left(t_{0}\right), d \boldsymbol{\mathcal { X }}\left(t_{0}\right)\right\rangle_{\mathcal{V}}+\int_{t_{0}}^{t_{f}}\left\langle\delta u(t),\left(\partial_{u} \mathbb{M}\right)^{*} \boldsymbol{\lambda}(t)\right\rangle_{U} d t
\end{aligned}
$$

where the adjoint of the tangent linear operators $\left(\partial_{\mathcal{X}} \mathbb{M}\right)^{*}$ : $\mathcal{V} \rightarrow \mathcal{V}$ and $\left(\partial_{u} \mathbb{M}\right)^{*}: \mathcal{V} \rightarrow U$ have been introduced. In order to get a simple and accessible solution for the cost function gradient, we impose that $\lambda\left(t_{f}\right)=0$ and that the adjoint variable $\boldsymbol{\lambda}$ is solution of the system:

$$
\left\{\begin{array}{l}
-\frac{\partial \boldsymbol{\lambda}}{\partial t}(t)+(\partial \mathcal{X} \mathbb{M})^{*} \boldsymbol{\lambda}(t)=(\partial \mathcal{X} \mathbb{H})^{*} R^{-1}(\mathcal{Y}-\mathbb{H}(\mathcal{X}))(t) \\
\boldsymbol{\lambda}\left(t_{f}\right)=0
\end{array}\right.
$$

Injecting now this relation into the cost function gradient (7), and using relation (9) we get (recalling that $d \mathcal{X}\left(t_{0}\right)=$ $\delta \boldsymbol{\epsilon}_{n}$ and $\left.d \mathcal{X}=(\partial \mathcal{X} / \partial u) \delta u(t)+\left(\partial \mathcal{X} / \partial \boldsymbol{\epsilon}_{n}\right) \delta \boldsymbol{\epsilon}_{n}\right)$ :

$$
\begin{aligned}
\left\langle\frac{\partial \mathcal{J}}{\partial \boldsymbol{\epsilon}_{n}}, \delta \boldsymbol{\epsilon}_{n}\right\rangle_{\mathcal{V}} & =-\left\langle\boldsymbol{\lambda}\left(t_{0}\right), \delta \boldsymbol{\epsilon}_{n}\right\rangle_{\mathcal{V}}+\left\langle B^{-1}\left(\boldsymbol{\mathcal { X }}\left(t_{0}\right)-\boldsymbol{\mathcal { X }}_{0}\right), \delta \boldsymbol{\epsilon}_{n}\right\rangle_{\mathcal{V}}, \\
\left\langle\frac{\partial \mathcal{J}}{\partial u}, \delta u\right\rangle_{U_{T}} & =\int_{t_{0}}^{t_{f}}\left\langle\delta u(t),\left(\partial_{u} \mathbb{M}\right)^{*} \boldsymbol{\lambda}(t)+F^{-1}\left(u(t)-u_{0}\right)\right\rangle_{U}^{d t} \\
& =\left\langle\left(\partial_{u} \mathbb{M}\right)^{*} \boldsymbol{\lambda}+F^{-1}\left(u-u_{0}\right), \delta u\right\rangle_{U_{T}} .
\end{aligned}
$$

From these relations, one can now readily identify the two components of the cost function derivatives with respect to the control variables:

$$
\begin{aligned}
& \frac{\partial \mathcal{J}}{\partial \boldsymbol{\epsilon}_{n}}=-\boldsymbol{\lambda}\left(t_{0}\right)+B^{-1}\left(\boldsymbol{\mathcal { X }}\left(t_{0}\right)-\boldsymbol{\mathcal { X }}_{0}\right), \\
& \frac{\partial \mathcal{J}}{\partial u}=\left(\partial_{u} \mathbb{M}\right)^{*} \boldsymbol{\lambda}+F^{-1}\left(u-u_{0}\right) .
\end{aligned}
$$

The partial derivatives of $\mathcal{J}$ are now simple to compute when the adjoint variable $\boldsymbol{\lambda}$ is available. Canceling out these derivatives leads to the following gradient descent algorithm:

$$
\begin{aligned}
\mathcal{X}\left(t_{0}\right) & =\mathcal{X}_{0}+B \boldsymbol{\lambda}\left(t_{0}\right), \\
u & =u_{0}+F\left(\partial_{u} \mathbb{M}\right)^{*} \boldsymbol{\lambda},
\end{aligned}
$$

where we introduced the pseudo-inverse $B$ and $F$ of $B^{-1}$ and $F^{-1}$. The adjoint variable is accessible through a forward integration of the state dynamics (1-2) and an backward integration of the adjoint variable dynamics (10).

The overall optimal control process can then be schematically summarized as follows:

1. Set initial conditions: $\mathcal{X}\left(t_{0}\right)=\mathcal{X}_{0}$ and $u=u_{0}$

2. From $\mathcal{X}\left(t_{0}\right)$, compute $\mathcal{X}(t)$ with the forward integration of relation (1)

3. Compute the adjoint variable $\boldsymbol{\lambda}(t)$ with the backward integration of system of equations (10)

4. Update the initial value $\mathcal{X}\left(t_{0}\right)$ and the parameter model $u$ with (12)

5. Loop to step 2 until convergence
Such a formulation provides us a practical framework for a tracking of very large state spaces. However, unless if a small temporal integration window of two frames is used [27], the approach is intrinsically a batch technique. The noise or the eventual missing of input data can easily be managed through the covariance matrix $R^{-1}$. In the following we exploit this formalism to perform the estimation of dense motion fields over a sequence of images.

\section{Application to Optical-Flow}

In this section, we propose to use this framework to compute dynamic consistent motion fields for two kinds of image sequences representing either fluid phenomenon or rigid objects. For fluid observations, the Navier-Stokes equations provide a reliable prior knowledge about their dynamics. The observation can be set to a simple measurement. Concerning the rigid objects, as no universal physical law can be stated, we rely on a coarser dynamical model associated to a more accurate observation based on a robust optical-flow formulation.

\subsection{Fluid sequences}

We are here interested in 1) a numerical simulation of an incompressible (i.e. divergence-free) 2D turbulence flow visualized through particles and 2) a cyclone observed from meteorological images. In both situations, for sake of clarity, we assume that the unknown velocity fields are divergence-free (solenoidal). Nevertheless, a divergent curl-free component (irrotational) driven by another coupled evolution law could also be handled $[12,25]$.

Dynamic model For this application we rely on the incompressible vorticity velocity formulation of the NavierStokes equation with an additive control variable, $u$. This variable aims at representing deviations from the pure vorticity transport model. These departures may be due to unknown forcing terms or to out of the plane motions. It allows us also dealing with compressible flows associated to low divergence value (intrinsically or at the observed scale). This model reads:

$$
\frac{\partial \xi}{\partial t}+\boldsymbol{v} \cdot \nabla \xi-\nu \Delta \xi=u
$$

The field $\boldsymbol{v}=\left(v_{x}, v_{y}\right)^{T}$ represents a solenoidal velocity field determined by its vorticity $\xi(\boldsymbol{v})=\partial v_{y} / \partial x-\partial v_{x} / \partial y$. The coefficient $\nu$ models the diffusion of the flow. The knowledge of $\xi$ enables to recover the motion field through BiotSavart law:

$$
\boldsymbol{v}=\nabla^{\perp} G * \xi,
$$

where $G$ denotes the Green kernel $\left(G=\frac{1}{2 \pi} \ln (|x|)\right)$ associated to the Laplacian operator. This computation can be very efficiently done in the Fourier domain [8].

Discretization and adjoint evolution model The discretization of the vorticity equation ought to be cautiously done. In particular, the advective term $\nabla \xi \cdot v$ must be treated 
specifically. In order to achieve an accurate and stable discretization of this term one must use conservative numerical schemes. Such schemes are designed to exactly respect the conservation law within the pixel by integrating the flux value at pixel boundaries. Total Variation Diminishing (TVD) scheme (which are Monotonicity preserving flux) prevents from an increase of oscillations over time and enables to transport shocks. In this work, we used semidiscrete central scheme $[15,16]$ associated to a second order accurate method [18] based on a Min-Mod limiter for the vorticity reconstruction [25]. The time integration is realized with a third-order Runge Kutta scheme, which also respect the TVD property [28].

The adjoint evolution model is constructed through a backward integration of the Runge-Kutta scheme [11] involving the same diffusive term (as the laplacian is auto adjoint) and the adjoint of the advective term (considering the velocity given by the forward integration of the vorticity transport equation). This latter term is as a consequence linear and its adjoint can be directly deduced from the adjoint of its discretization.

Observation Operator Starting on the well-known optical flow constraint equation, one can assume, in order to cope with the aperture problem, that if the unknown optic flow vector at a location $\boldsymbol{x}$ is constant within some neighborhood of size $n$ [20], the motion field is obtained by minimizing:

$$
K_{n} *\left(\frac{\partial E(\boldsymbol{x}, t)}{\partial t}+\nabla E(\boldsymbol{x}, t) \cdot \boldsymbol{v}(\boldsymbol{x}, t)\right)^{2},
$$

where $E$ stands for the luminance function and $K_{n}$ is a Gaussian kernel of standard deviation $n$. This LucasKanade formulation yields to the relation:

$$
K_{n} *\left[\begin{array}{cc}
E_{x}^{2} & E_{x} E_{y} \\
E_{x} E_{y} & E_{y}^{2}
\end{array}\right] \boldsymbol{v} \approx-K_{n} *\left[\begin{array}{c}
E_{x} E_{t} \\
E_{y} E_{t}
\end{array}\right],
$$

where $E_{\bullet}=\partial E(\boldsymbol{x}, t) / \partial \bullet$. In our application, the system's state is $\xi(t)$ connected to $\boldsymbol{v}$ through relation (14). As a consequence, our observation system reads :

$$
\begin{gathered}
\mathcal{Y}=-\left[\begin{array}{c}
K_{n} *\left(E_{x} E_{t}\right) \\
K_{n} *\left(E_{y} E_{t}\right)
\end{array}\right] \\
\text { and } \mathbb{H}(\xi(t), t)=\left[\begin{array}{cc}
K_{n} *\left(E_{x}^{2}\right) & K_{n} *\left(E_{x} E_{y}\right) \\
K_{n} *\left(E_{x} E_{y}\right) & K_{n} *\left(E_{y}^{2}\right)
\end{array}\right] \nabla^{\perp} G * \xi(t) .
\end{gathered}
$$

This observation operator is also linear w.r.t the state's variable $\xi$. Therefore, its linear tangent operator is itself. The expression of its adjoint is not trivial as this operator is expressed through a convolution product. It can be demonstrated in the Fourier space that its expression is $\left(\partial_{\xi} \mathbb{H}\right)^{*}=$ $-\mathbb{H}$. Finally, let us note that, as the dynamic model is quite accurate, the initial field was set to zero $\left(\boldsymbol{v}_{0}(\boldsymbol{x})=0\right)$. To deal with the larger displacements, a multi-resolution approach has been used.

Covariances The covariance matrix of the initial condition $B$ and the covariance matrix of the parameter $Q$ have been fixed to constant diagonal matrices.
The matrix $R$ linked to the observations is defined as:

$$
R=C\left(I d-e^{-|\nabla E(\boldsymbol{x}, t)| / \sigma^{2}}\right),
$$

where $C$ and $\sigma$ are parameters to be fixed. When no observation is available, this matrix is set to infinity. In all our applications, we fixed $B=0.1, Q=0.001, \sigma=0.9$ and $C \in[50 ; 500]$.

Situation \#1 : Synthetic DNS fluid motion sequence This first sequence of 50 frames has been obtained from a numerical simulation of an incompressible 2D turbulence flow with an unknown forcing term (at Reynolds number, $R e=1 / \nu=4000$ ). This sequence simulates an experimental flow seeded with particles. Such kind of data are largely used in the experimental fluid mechanics community. The top of figure 1 represents: a PIV image (a), a motion field estimated in the sequence (b), two synthetic maps of vorticity $(e, f)$ and the corresponding estimated maps $(g, h)$. It can be observed that the extracted motion fields are in accordance with the ground truth. In addition, we present two quantitative evaluations of our motion fields compared to the ground truth and to the ones obtained with a fluid dedicated motion estimator [7] in (c,d). These comparisons are made on the basis of the vorticity and a spectral analysis on the row average. These two comparisons prove the ability of our approach to recover very accurate motion fields with a better estimation of small scales structures which are smoothed out by the dense fluid motion estimator [7].

Situation \#2 : Cyclone Vince sequence We applied this technique on an infra-red meteorological sequence of 17 frames showing the Vince cyclone over north Atlantic. The sequence was acquired on the 9 October 2005. The top of figure 2 presents the estimated motion fields superimposed to their corresponding image for three frames of the sequence. The second line presents the corresponding vorticity and on the third line we plotted the vorticity obtained with a dedicated fluid motion estimator [7]. As many location of the image plane are disturbed by non-valid data, it can be observed that the dedicated estimator presents some temporal inconsistencies and discontinuities in terms of vorticity. These noisy values are completely removed by the technique presented which provides smooth and coherent vorticity maps.

\subsection{Video sequence}

We tested our approach on the 10 first images of the wellknown taxi sequence. To assess the benefit of the temporal model, we blurred 3 images as depicted in figure $3(b-d)$. This kind of artifacts may appear when, for instance, one wants to restore old videos.

Dynamic model No universal physical law can be stated for general videos showing moving objects of different natures. It is therefore difficult to propose a generic formula- 


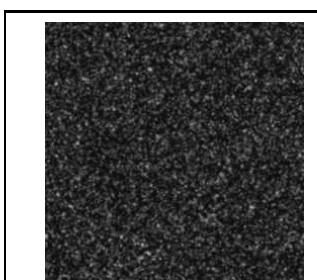

(a)

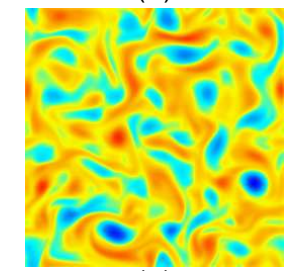

(e)

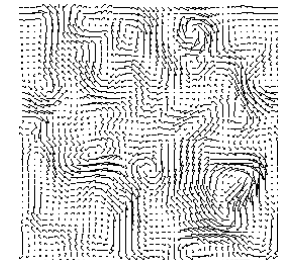

(b)

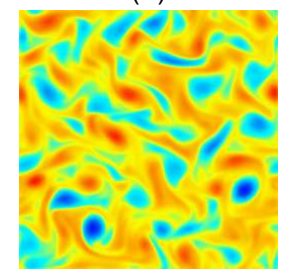

(f)

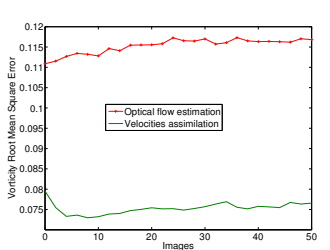

(c)

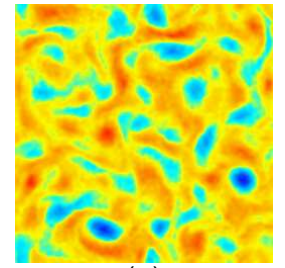

(g)

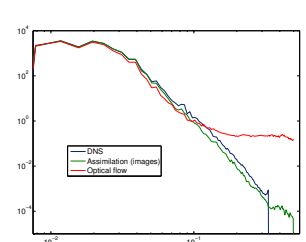

(d)

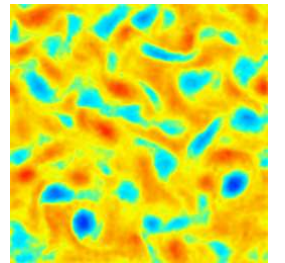

(h)

Figure 1. Experimental results on DNS: Sequence \#1: (a): a PIV image; (b): an estimated motion field of the sequence; (c): the vorticity error compared to an optical-flow estimation; (d): spectra of the velocities, $(e, f)$ : two maps of vorticity issued from DNS and $(\mathrm{g}, \mathrm{h})$ : the corresponding estimated maps.

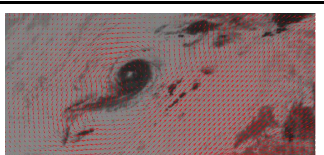

(a)

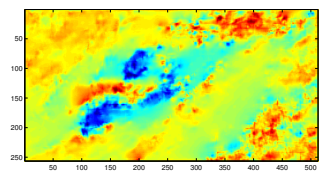

(d)

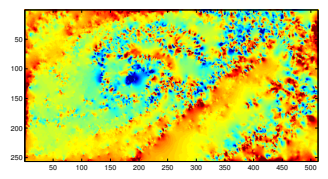

(g)

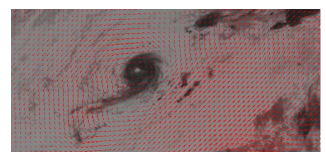

(b)

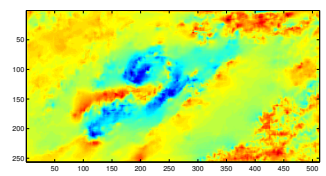

(e)

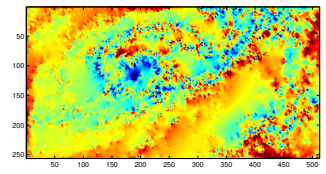

(h)

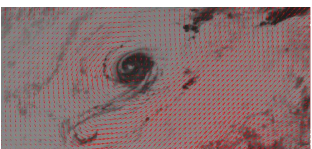

(c)

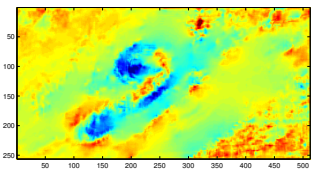

(f)

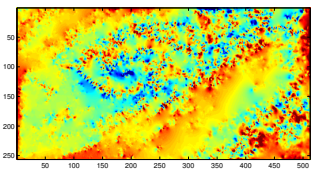

(i)

Figure 2. Experimental results on Vince sequence: Sequence \#2: $(a-c)$ : three images and the corresponding motion field; (d-f): the estimated vorticity; $(\mathrm{g}-\mathrm{i})$ : the vorticity obtained with a fluid dedicated estimator without any temporal consistency.

tion that describes accurately the evolution of moving objects. However, one can rationally assume over a short range of time that the velocity is transported by itself up to a Gaussian discretization error. Defining the location $\boldsymbol{x}_{t}$ of a point as a stochastic process driven by an Ito diffusion $d \boldsymbol{x}_{t}=\boldsymbol{v}\left(\boldsymbol{x}_{t}\right)+\nu d B$ and noting $g=\mathbb{E}\left[f\left(x_{t} \mid x_{t-h}\right)\right]$, the expectation of a function of $\boldsymbol{x}_{t}$ given $\boldsymbol{x}_{t-h}$ reads, through the forward Kolmogorov equation, $\partial g / \partial t=-\nabla g \cdot \boldsymbol{v}+1 / 2 \nu^{2} \Delta g$ [24]. We therefore assume the following dynamical model:

$$
\frac{d \boldsymbol{v}}{d t}=\frac{\partial \boldsymbol{v}}{\partial t}+\nabla \boldsymbol{v} \cdot \boldsymbol{v}=\nu \Delta \boldsymbol{v}
$$

where $\nu$ is to be fixed, but could be considered as a model's parameter. The operator $\mathbb{M}$ is then:

$$
\mathbb{M}(\boldsymbol{v}(\boldsymbol{x}, t))=\boldsymbol{v}(\boldsymbol{x}, t) \cdot \boldsymbol{\nabla} \boldsymbol{v}(\boldsymbol{x}, t)-\nu \Delta \boldsymbol{v}(\boldsymbol{x}, t) ;
$$

the associate tangent linear operator at point $\boldsymbol{v}$ for a perturbation $\delta \boldsymbol{v}$ is

$$
\left(\partial_{v} \mathbb{M}\right) \delta \boldsymbol{v}=\boldsymbol{v} \cdot \boldsymbol{\nabla} \delta \boldsymbol{v}+\delta \boldsymbol{v} \cdot \boldsymbol{\nabla} \boldsymbol{v}-\nu \Delta \delta \boldsymbol{v},
$$

and its adjoint $\left(\partial_{v} \mathbb{M}\right)^{*}$ used in (10) is obtained by the transpose of the matrix corresponding to the linear tangent operator discretization.

Observation Operator As no accurate dynamic model is available in that case, we prefer to apply a more suitable observation operator based on the optical flow constraint equation accompanied with a first order robust smoothing. The motion field $v$ to extract may be formulated as:

$$
\begin{aligned}
\boldsymbol{v}(\boldsymbol{x}, t) \approx & \min _{\boldsymbol{v}(\boldsymbol{x}, t)} \int_{\Omega} f_{1}\left(\left[\boldsymbol{\nabla} E(\boldsymbol{x}, t) \cdot \boldsymbol{v}(\boldsymbol{x}, t)+E_{t}(\boldsymbol{x}, t)\right]^{2}\right) \\
& +\alpha \int_{\Omega} f_{2}\left(\left|\boldsymbol{\nabla} v_{x}\right|^{2}\right)+f_{2}\left(\left|\boldsymbol{\nabla} v_{y}\right|^{2}\right)
\end{aligned}
$$


where $\Omega$ is the image plane, $\alpha$ is a smoothing positive parameter and $f_{1}$ and $f_{2}$ are two robust penalty functions [3] such that $f\left(\sqrt{y^{2}}\right)$ is concave. A weighted quadratic formulation of these cost functions can then be obtained [10]:

$$
\begin{aligned}
& \boldsymbol{v}(\boldsymbol{x}, t) \approx \min _{\boldsymbol{v}, \delta_{o}, \delta_{u}, \delta_{v}} \int_{\Omega} \delta_{o}\left[\boldsymbol{\nabla} E(\boldsymbol{x}, t) \cdot \boldsymbol{v}(\boldsymbol{x}, t)+E_{t}(\boldsymbol{x}, t)\right]^{2} \\
& +\phi\left(\delta_{o}\right)+\alpha \int_{\Omega} \delta_{u}(|\boldsymbol{\nabla} u|)^{2}+\delta_{v}(|\boldsymbol{\nabla} v|)^{2}+\phi\left(\delta_{u}\right)+\phi\left(\delta_{v}\right),
\end{aligned}
$$

where the minimization w.r.t the additional outliers variables $\left(\delta_{\bullet}\right)$ is given explicitly through the derivative of function $f: \hat{\delta}_{\bullet}(y)=f_{\bullet}^{\prime}\left(\sqrt{y^{2}}\right)$ (see [13] for more details). The Euler-Lagrange equations of the previous functional may be written as the following system of coupled equations:

$$
\begin{aligned}
& \delta_{o} \boldsymbol{\nabla} E(\boldsymbol{x}, t)\left(\boldsymbol{\nabla} E(\boldsymbol{x}, t)^{T} \boldsymbol{v}(\boldsymbol{x}, t)+E_{t}(\boldsymbol{x}, t)\right)- \\
& \alpha \delta_{u} \Delta \boldsymbol{\nabla} u-\alpha \delta_{v} \Delta \boldsymbol{\nabla} v=0 \text { and } \delta_{\bullet}(x)=f_{\bullet}^{\prime}\left(\sqrt{x^{2}}\right) .
\end{aligned}
$$

The observation system for our tracking problem is thus defined as (with $\delta_{\bullet}(x)=f_{\bullet}^{\prime}\left(\sqrt{x^{2}}\right)$ ):

$$
\begin{aligned}
& \mathbb{H}(\boldsymbol{v}(t), t)=\left[\delta_{o} \boldsymbol{\nabla} E(\boldsymbol{x}, t) \boldsymbol{\nabla} E(\boldsymbol{x}, t)^{T}-\alpha\left(\begin{array}{cc}
\delta_{u} \Delta & 0 \\
0 & \delta_{v} \Delta
\end{array}\right)\right] \boldsymbol{v}(t) \\
& \boldsymbol{Y}=-\delta_{o} \boldsymbol{\nabla} E(\boldsymbol{x}, t) E_{t}(\boldsymbol{x}, t)
\end{aligned}
$$

Given the weight values (which are obtained explicitly for a value of the velocity and the corresponding robust function argument), the operator $\mathbb{H}$ is linear w.r.t. the state function $\boldsymbol{v}$. Its tangent linear operator is itself and the adjoint operator $\left(\partial_{\boldsymbol{v}} \mathbb{H}\right)^{*}$ used in relation (10) is given by the transpose of the matrix corresponding to its discretization. As for the initial field it has been set to a motion field recovered from a fast Horn \& Schunck estimator embedded into a multiresolution framework. This initial field is then propagated through the considered dynamics and can be seen as a first coarse estimation. The assimilation process is furthermore much faster than a sequence of motion estimations.

Results The figures in $3(\mathrm{e}-\mathrm{h})$ represent 4 motion fields obtained with our approach whereas figures $3(i-I)$ show the corresponding motion fields obtained with a robust motion estimator [21]. Thanks to the optimal control process, the motion fields recovered are not affected by the strong artifacts introduced. This kind of results might be interesting for applications in the field of video coding and/or restoration. As for the computation time, this process was twotime faster than the successive estimations of dense motion fields with the same observation operator. Such an approach could also be used to estimate motion of temporarily occluded objects in video, and as a consequence constitutes an important basic ingredient for video object removing in video post-processing.

\section{Conclusion}

In this paper a framework allowing us to estimate and track dense motion fields with a temporal consistency has been introduced. The approach is related to optimal control theory. It authorizes through noisy and incomplete observations to estimate a sequence of motion fields guided by a dynamical model. To correctly deal with the huge state space we are facing, the minimization is handled using an adjoint formulation. The resulting process consists in alternating a forward integration of the state variable and a backward integration of the introduced adjoint variables.

This technique has been successfully applied and compared with usual optical flows estimators in three different situations: a fluid motion simulation, a real fluid sequence depicting a cyclone and the real taxi sequence. Concerning fluid images, the results are very good despite the fact that no information of the initial field was assumed. For the rigid motion example, the definition of the dynamical model is more complex since no accurate law can be defined in such a situation. Hence, we rely on a more sophisticated observations and an initial motion field based on a Horn \& Schunck estimation. The rest of the process is then very fast and the quality of the recovered motion fields is good and consistent in time despite the artifacts introduced.

Acknowledgments This work was supported by the European Community through the IST FET Open FLUID Project (http://fluid.irisa.fr)

\section{References}

[1] L. Alvarez, J. Weickert, and J. Sànchez. Reliable estimation of dense optical flow fields with large displacements. Int. J. of Comp. Vis., 39(1):41-56, 2000.

[2] J. Barron, D. Fleet, and S. Beauchemin. Performance of optical flow techniques. Int. J. of Com. Vis., 12(1):43-77, 1994.

[3] M. Black. Recursive non-linear estimation of discontinuous flow fields. In Proc. Eur. Conf. on Comp. Vis., pages 138145, Stockholm, Sweden, 1994.

[4] M. Black and P. Anandan. Robust dynamic motion estimation over time. In IEEE Conf. on Comp. Vis. and Patt. Rec., pages 292-302, Maui, HI, USA, 1991.

[5] T. Brox, A. Bruhn, N. Papenberg, and J. Weickert. High accuracy optical flow estimation based on a theory for warping. In Eur. Conf. On Com. Vis., volume 4, pages 25-36, 2004.

[6] A. Bruhn, J. Weickert, T. Kohlberger, and C. Schnoerr. A multigrid platform for real-time motion computation with discontinuity-preserving variational methods. Int. J. Com. Vis., 70(3):257-277, 2006.

[7] T. Corpetti, E. Mémin, and P. Pérez. Dense estimation of fluid flows. IEEE T. on Patt. Ana. and Mach. Int., 24(3):365380, March 2002.

[8] T. Corpetti, E. Mémin, and P. Pérez. Extraction of singular points from dense motion fields: an analytic approach. J. of Math. Im. and Vis., 19(3):175-198, 2003.

[9] B. Galvin, B. McCane, K. Novins, D. Mason, and S. Mills. Recovering motion fields: an analysis of eight optical flow algorithms. In Brit. Mach. Vis. Conf., Southampton, 1998.

[10] D. Geman and G. Reynolds. Constrained restoration and the recovery of discontinuities. Pat. Ana. Mach. Int., 14(3):367383, 1992. 


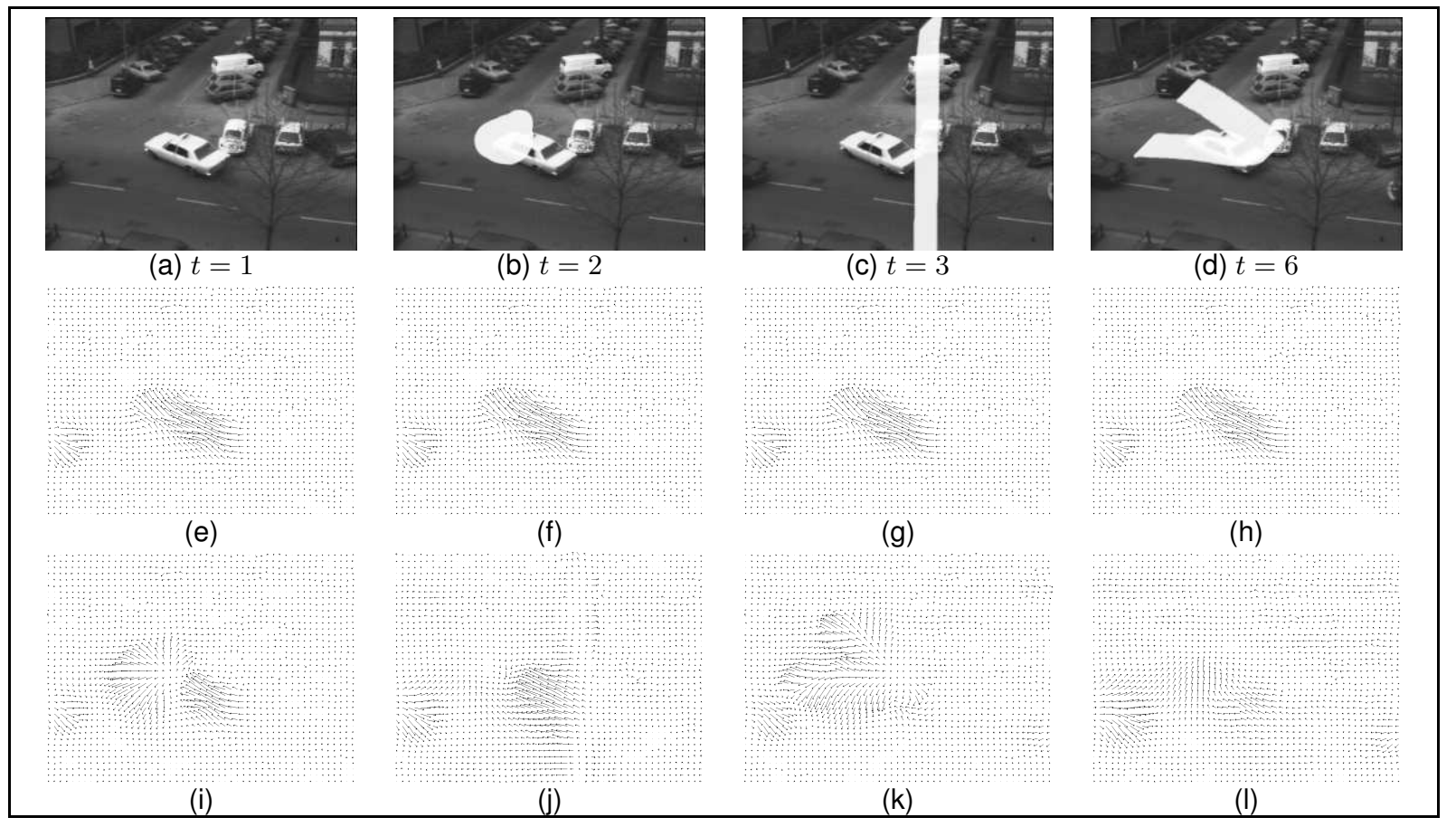

Figure 3. Experimental results: Sequence \#3: (a): initial image; $(b-d)$ : the three blurred images of the sequence; (e-h) measured motion fields between times $(t=1, t=2),(t=2, t=3),(t=5, t=6)$ and $(t=6, t=7)$ respectively with our approach; (i-l) the corresponding motion fields obtained without a temporal consistency.

[11] M. Giles. On the use of runge-kutta time-marching and multigrid for the solution of steady adjoint equations. Technical Report 00/10, Oxford Univ. Comp. Lab., 2000.

[12] P. Heas, E. Mémin, N. Papadakis, and A. Szantai. Layered estimation of atmospheric mesoscale dynamics from satellite imagery. IEEE T. Geoscience and Remote Sensing, 2007.

[13] P. Holland and R. Welsch. Robust regression using iteratively reweighted least-squares. Commun. Statis.-Theor. Meth., A6(9):813-827, 1977.

[14] B. Horn and B. Schunck. Determining optical flow. Artificial Intelligence, 17:185-203, 1981.

[15] A. Kurganov and D. Levy. A third-order semi-discrete central scheme for conservation laws and convection-diffusion equations. SIAM J. on Sci. Comp., 22(4):1461-1488, 2000.

[16] A. Kurganov and E. Tadmor. New high resolution central schemes for nonlinear conservation laws and convectiondiffusion equations. J. of Comp. Physics, 160(1):241-282, 2000.

[17] F. Le-Dimet and O. Talagrand. Variational algorithms for analysis and assimilation of meteorological observations: theoretical aspects. Tellus, pages 97-110, 1986.

[18] D. Levy and E. Tadmor. Non-oscillatory central schemes for the incompressible 2d-euler equations. Math. Res. Let., 4:321-340, 1997.

[19] J. Lions. Optimal Control of Systems Governed by Partial Differential Equations. Springer-Verlag, 1971.

[20] B. Lucas and T. Kanade. An iterative image registration technique with an application to stereo vision. In Int. Joint Conf. on Art. Int., pages 674-679, Vancouver, Canada, 1981.
[21] E. Mémin and P. Pérez. Hierarchical estimation and segmentation of dense motion fields. Int. J. of Comp. Vis., 46(2):129-155, 2002.

[22] H. Nagel. Extending the oriented smoothness constraint into the temporal domain and the estimation of derivatives of optical flow. In Eur. Conf. Com. Vis., pages 139-148, 1990.

[23] P. Nesi. Variational approach to optical flow estimation managing discontinuities. Im. \& Vis. Comp., 11(7):419-439, 1993.

[24] B. Oksendal. Stochastic differential equations. SpingerVerlag, 1998.

[25] N. Papadakis and E. Mémin. A variational framework for spatio-temporal smoothing of fluid motions. In Scale Space and Variational Methods, Ischia, Italy, 2007.

[26] N. Papenberg, A. Bruhn, T. Brox, S. Didas, and J. Weickert. Highly accurate optic flow computation with theoretically justified warping. Int. J. of Comp. Vis., 67(2):141-158, April 2006.

[27] P. Ruhnau and C. Schnoerr. Optical stokes flow estimation: An imaging-based control approach. Exp.in Fluids, 42(1):61-78, 2007.

[28] C. Shu. Advanced Numerical Approximation of Nonlinear Hyperbolic Equations, Lecture Notes in Mathematics, chapter Essentially non-oscillatory and weighted essentially nonoscillatory schemes for hyperbolic conservation laws, pages 325-432. Springer, 1998.

[29] J. Weickert and C. Schnörr. Variational optic flow computation with a spatio-temporal smoothness constraint. J. of Math. Im. and Vis., 14(3):245-255, 2001. 